\section{Collectible optical power of various specially shaped multimode optical fiber probes for contact sensing}

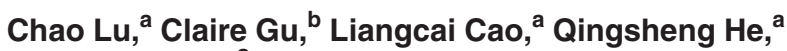 \\ and Guofan Jin $^{\mathrm{a}}$ \\ ${ }^{a}$ Tsinghua University, State Key Laboratory of Precision \\ Measurement Technology and Instruments, Beijing, \\ 100084; China \\ E-mail: Ichao.thu@gmail.com \\ b University of California, Department of Electrical \\ Engineering, Santa Cruz, California 95064
}

\begin{abstract}
We present a theoretical analysis of the total collectible optical power of an arbitrarily shaped multimode optical fiber probe for contact sensing where the signal from the analyte is emitted or scattered on the outside surface of the fiber tip. Calculation results and optimization parameters are given for spherical, parabolic, and cone-shaped fiber probes. This analysis can be used to guide the design and optimization of various fiber sensors, including fluorescence, surface plasmon resonance, and surface enhanced Raman sensors. ( 2008 Society of Photo-Optical Instrumentation Engineers. [DOI: 10.1117/1.2829151]
\end{abstract}

Subject terms: fiber optics sensors; geometrical optics; optical design; sensors.

Paper 070293LR received Apr. 25, 2007; revised manuscript received Sep. 26, 2007; accepted for publication Nov. 3, 2007; published online Jan. 8, 2008.

\section{Introduction}

In single-fiber sensors, where a fiber is used to transmit both the excitation light and the signal from the analyte, ${ }^{1,2}$ the signal from the analyte is often emitted or scattered on the outside surface of the fiber tip, i.e., the sample is in contact with the fiber probe, known as contact sensing. To increase the sensitivity of these sensors, it is desirable to increase the active surface area where the signal is generated and collected and to shape the probe in such a geometry that most of the signal light can be coupled into the transmission (confined) modes of the fiber. While multimode fibers can be used to increase the active surface area, it is not clear how the geometry of the fiber probe affects the total collectible optical power. In this paper, we analyze the total collectible optical power of various specially shaped multimode optical fiber probes for contact sensing. With the examples of spherical, parabolic, and cone-shaped fiber tips, we show that an optimal geometry exists for each type of end-polished fiber probes. This analysis can be used to guide the design and optimization of various fiber sensors, including fluorescence sensors, ${ }^{3}$ surface plasmon resonance sensors, ${ }^{4}$ surface enhanced Raman sensors, ${ }^{1,2}$ etc.

\section{Theoretical Model}

The coupling efficiency from a source to an optical fiber has been calculated using geometrical approaches. ${ }^{5}$ How-

0091-3286/2008/\$25.00 @ 2008 SPIE

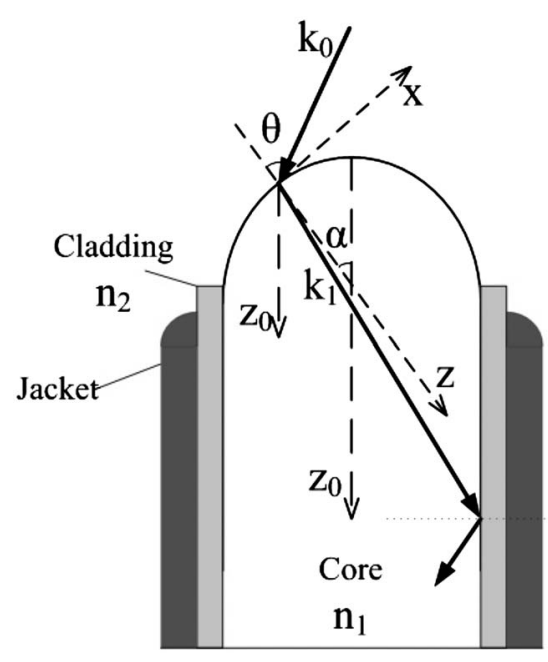

Fig. 1 Schematic diagram of a spherical-tip fiber probe consisting of a core, a cladding, and a protection jacket. $\mathbf{k}_{0}$ is the wave vector of the incident light, and $\theta$ is the incidence angle in air. After refraction, the ray is coupled into the fiber and transmitted by the total internal reflection. $(x, y, z)$ is the local coordinate system, where $\mathbf{z}$ is normal to the local surface, $\mathbf{z}_{0}$ is the direction of the fiber axis, and $\alpha$ is the angle between $\mathbf{z}$ and $\mathbf{z}_{0}$.

ever, most of the calculations on the shape-dependence of the fiber were calculated numerically using certain software, such as ZEMAX.

Consider a multimode fiber with its end polished to a well-defined (e.g., spherical) shape where the signal light is generated and coupled into the fiber (see Fig. 1). As the core diameter of the fiber is much larger than the wavelength of light, we use a geometrical optics model to study the total collectible optical power. ${ }^{5}$ For a long enough fiber, only those rays that satisfy the total internal reflection condition at the interface between the core and the cladding of the fiber can successfully reach the detector.

For a fiber probe with a specially shaped tip, we calculate the total collectible optical power as follows. Pick an arbitrary point on the fiber surface. Let $(x, y, z)$ be the local coordinate system, where the $z$ direction is normal to the local surface. In the case of a cylindrically symmetric fiber, such as a spherical one and the ones discussed later in this paper, we can have both the $x$ and $z$ axes in the crosssectional plane containing the fiber axis. Consider an incident ray with the wave vector $\mathbf{k}_{\mathbf{0}}$ in air. In a local spherical coordinate system $(r, \theta, \varphi), \mathbf{k}_{\mathbf{0}}$ is written as

$k_{0 x}=k_{0} \sin \theta \cos \varphi, \quad k_{0 y}=k_{0} \sin \theta \sin \varphi$,

where $\theta$ is the incidence angle, and $\varphi$ is the azimuth angle. After refraction, inside the fiber, the wave vector becomes $\mathbf{k}_{1}$. According to Snell's Law, $k_{1 x}=k_{0 x}, k_{1 y}=k_{0 y}$, and

$k_{1 z}=\left[\left(\frac{2 \pi n_{1}}{\lambda}\right)^{2}-k_{1 x}^{2}-k_{1 y}^{2}\right]^{1 / 2}=\left[\left(\frac{2 \pi n_{1}}{\lambda}\right)^{2}-k_{0}^{2} \sin ^{2} \theta\right]^{1 / 2}$,

where $n_{1}$ is the refractive index of the core.

If the angle between $\mathbf{k}_{\mathbf{1}}$ and $z_{0}$ is less than $\theta_{\max }$, then this ray is confined inside the fiber core, where $\theta_{\max }$ is $(\pi / 2)$ $-\theta_{c}$, with $\theta_{c}$ the critical angle for the total internal reflection at the interface between the fiber core and cladding. 
Supposing that the refractive indices of the core and the cladding are $n_{1}$ and $n_{2}$, respectively, the confined rays must satisfy the following condition:

$\mathbf{k}_{1} \cdot \mathbf{z}_{0} \geqslant \frac{2 \pi n_{1}}{\lambda} \cos \theta_{\max }=\frac{2 \pi n_{1}}{\lambda} \cdot \frac{n_{2}}{n_{1}}=\frac{2 \pi n_{2}}{\lambda}$.

Let $\alpha$ be the angle between $z_{0}$ and $z$. In the $(x, y, z)$ coordinate, the unit vector in the $z_{0}$ direction can be written as $\hat{z}_{0}=(\sin \alpha, 0, \cos \alpha)$, and Eq. (2) becomes:

$k_{1 z}=k_{1 x} \sin \alpha+k_{1 z} \cos \alpha \geqslant \frac{2 \pi n_{2}}{\lambda}$.

Substituting Eq. (1) and the expression of $k_{1 z}$ into Eq. (3), we can deduce:

$\sin \theta \cos \phi \sin \alpha+\left(n_{1}^{2}-\sin ^{2} \theta\right)^{1 / 2} \cos \alpha \geqslant n_{2}$.

So the optical power collectible at this point and transmittable through the fiber is:

$p(\alpha, \beta)=\iint T(\theta, \varphi) \sin \theta \mathrm{d} \theta \mathrm{d} \varphi$,

where $T(\theta, \varphi)$ is the radiance from an individual emitting/ scattering point source on the surface of the fiber transmitted into the fiber core (with the consideration of the loss due to surface reflection), and the integration is carried out under the restriction of Eq. (4). With this restriction, only the light that satisfies the total internal reflection condition is included in the calculation; therefore, the collectible power indicates the portion of power that can reach the output end of the fiber. In general, the collectible power at certain point of the fiber tip may be a function of both the angle $\alpha$ and the azimuth angle $\beta$ in the global $\left(x_{0}, y_{0}, z_{0}\right)$ coordinate system. In the case of a cylindrically symmetric fiber, it is only a function of $\alpha$. Intuitively, the acceptance solid angle [integration limit in Eq. (5)] corresponds to the overlap between two solid angles. One of them is the solid angle containing all possible confined rays, and the other is the solid angle containing all the refracted rays entering the surface at the given point. The total optical power collectible over the entire probe surface area is then:

$$
\begin{aligned}
P= & \iint p\left[x_{0}, y_{0}, z_{0}\left(x_{0}, y_{0}\right)\right] \mathrm{d} S=\iint p\left[x_{0}, y_{0}, z_{0}\left(x_{0}, y_{0}\right)\right] \\
& \times\left(1+z_{0 x}^{\prime 2}+z_{0 y}^{\prime 2}\right)^{1 / 2} \mathrm{~d} x_{0} \mathrm{~d} y_{0},
\end{aligned}
$$

where $p\left[x_{0}, y_{0}, z_{0}\left(x_{0}, y_{0}\right)\right]$ is the same as $p(\alpha, \beta)$ in Eq. (5), rewritten in the global Cartesian coordinate system $\left(x_{0}, y_{0}, z_{0}\right) ; z_{0}\left(x_{0}, y_{0}\right)$ is the equation that describes the curved surface of the fiber tip; and $z_{0 x}^{\prime}$ and $z_{0 y}^{\prime}$ are partial derivatives of $z_{0}\left(x_{0}, y_{0}\right)$ with respect to $x_{0}$ and $y_{0}$, respectively. The integration is carried out over the surface area that is in contact with the emitting/scattering source. Notice that the choice of the origin of the global coordinate system is arbitrary. In the case of a spherical fiber tip, it can be conveniently chosen as the center of the sphere so that the integration can be calculated in the spherical coordinate system, $P=2 \pi \int_{0}^{\alpha_{\max }} p(\alpha) R^{2} \sin \alpha \mathrm{d} \alpha$, where $R$ is the radius of the sphere, and $\alpha_{\max }$ is the maximum angle between the

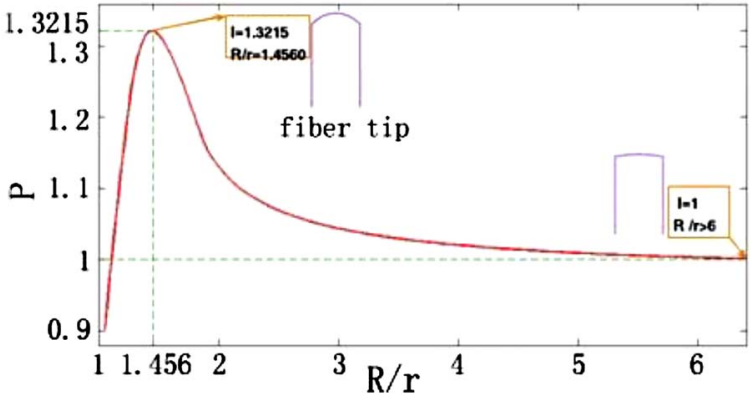

Fig. 2 Total collectible optical power as a function of $R / r$, where $r$ is the radius of the fiber core, and $R$ is the radius of the sphere at the end of the fiber. The collectible optical power of a flat-tip fiber with $R \gg r$ is normalized to 1 and taken as the reference.

$\mathbf{z}$ and $\mathbf{z}_{\mathbf{0}}$ axes. In the case of other shapes, such as parabolic or cone-shaped fiber tips, Eq. (6) can be used in numerical calculations.

\section{Results for Fiber Probes with Three Different Geometrical Shapes}

Using typical multimode fiber parameters, i.e., the diameter of the core $D=62.5 \mu \mathrm{m}$ and the refractive indices of the core and cladding $n_{1}=1.4800$ and $n_{2}=1.4546$, the total optical power collectible by spherical, parabolic, and coneshaped fiber tips are calculated and discussed in the following.

For a fiber probe with a spherical tip, the calculation result of the total collectible optical power [Eq. (6)] is shown in Fig. 2. For simplicity, we have neglected any energy loss due to reflection, scattering, or absorption when the rays enter the fiber. Such loss can be included with the knowledge of the external medium. Furthermore, we have also assumed that each point on the outside surface of the fiber tip acts as a point source. This assumption can be modified with the knowledge of the specific radiation pattern of the light emitting/scattering process involved in the sensing mechanism.

Notice that when $R \gg r$, the end surface becomes a flat plane. In this case, the active area where the signal is generated is the smallest compared with fibers having other shaped tips; however, the acceptance solid angle at any point is the largest. As the $R / r$ value decreases, the active surface area increases; however, the acceptance solid angle decreases as we move away from the fiber axis. Figure 2 shows the collectible optical power for various radii of curvature normalized to that of a flat end. The results are obtained by numerically integrating Eq. (6) using MATLAB. As shown in Fig. 2, when $R / r$ equals 1.4560 , the collectible optical power reaches its maximum, which is $45.60 \%$ higher than that collectible by a fiber with a flat end.

The total collectible optical power using parabolic fiber tips is shown in Fig. 3. Again, using the flat tip as the reference, the total collectible optical power reaches its maximum when $d=0.008$, i.e., the expression of the parabola is $z=-0.016 x^{2}$. As $d$ increases further, the tip of the fiber becomes sharper and the collectible optical power becomes smaller due to the decreased acceptance solid angles at points other than the vertex. For parabolic-shaped fiber 


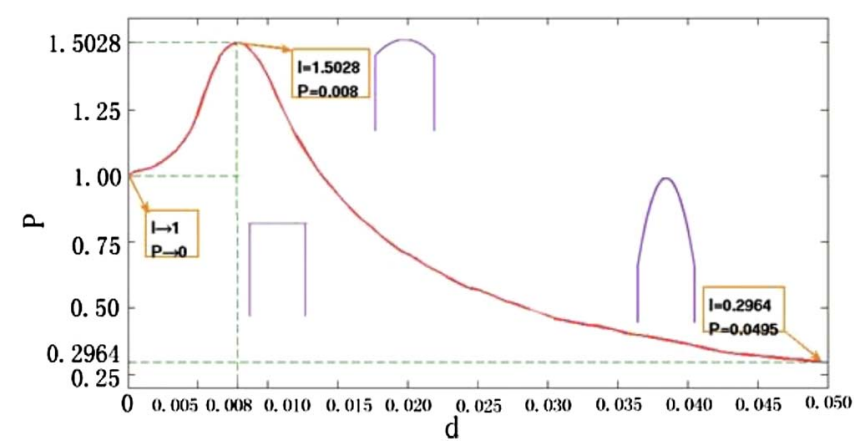

Fig. 3 Total collectible optical power as a function of $d$, the distance between the vertex and the focus of the parabola representing the sharpness of the parabolic probe. When $d$ equals 0 , the shape of the end becomes flat, and the corresponding power is taken as the normalized reference.

probes, the maximum collectible optical power is $50.28 \%$ higher than that collectible with a flat-tip probe.

The total collectible optical power as a function of the half vertex angle of a cone-shaped fiber tip is shown in Fig. 4. When the half vertex angle of the cone tip is smaller than $36.899 \mathrm{deg}$, there is no collectable signal, because none of the rays entering from the side walls are confined. Notice that this is a result of the geometrical optics approximation for multimode fibers, which is no longer valid at the tip of a slowly tapered fiber. In this region, more accurate analysis needs to be carried out with the modal calculation of a tapered fiber. The total collectible optical power reaches its maximum, which is $119 \%$ larger than that collectible by a flat-ended fiber, when the half vertex angle equals $54.43 \mathrm{deg}$.

\section{Conclusion}

In summary, for contact sensing using an end-polished multimode optical fiber probe, we have developed a physical

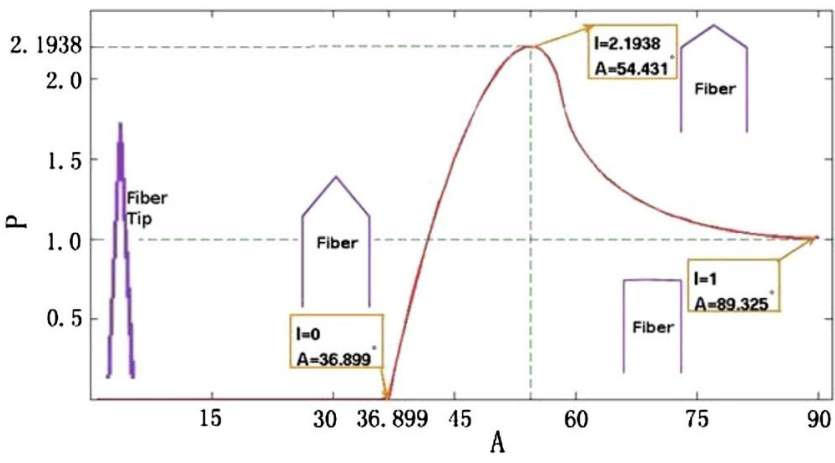

Fig. 4 Total collectible optical power as a function of $A$, the halfvertex angle of the cone representing the sharpness of the fiber tip. The total optical power collectible by a flat-ended fiber probe is taken as 1 .

model to calculate and optimize the total collectible optical power using fiber tips with various geometrical shapes. The optimization parameters for fiber probes with spherical, parabolic, and cone-shaped tips are given. Compared to a flat-ended fiber probe, our results show that the total collectible optical power can be improved by a factor of 1.3 to 2.2 depending on the shape of the tips.

\section{References}

1. Y. Zhang, C. Gu, A. M. Schwartzberg, and J. Z. Zhang, "Surfaceenhanced Raman scattering sensor based on D-shaped fiber," Appl. Phys. Lett. 87, 123105 (2005).

2. D. L. Stokes and T. Vo-Dinh, "Development of an integrated singlefiber SERS sensor," Sens. Actuators B 69, 28-36 (2000).

3. N. E. Marcon and B. C. Wilson, "The value of fluorescence techniques in gastrointestinal endoscopy better than the endoscopist's eye? II: the North American experience," Endoscopy 30(4), 419-421 (1998).

4. R. C. Jorgenson and S. S. Yee, "A fiber-optic chemical sensor based on surface plasmon resonance," Sens. Actuators B 12, 213-220 (1993).

5. Gerd Keiser, Optical Fiber Communications, 3rd ed., McGraw-Hill, Boston (2000). 